\title{
MicroRNA-155-5p inhibits the invasion and migration of prostate cancer cells by targeting SPOCK1
}

\author{
LIN-YA YAO ${ }^{1,2}$, JUN MA $^{2}$, XUE-MING ZENG ${ }^{2}$ and JUN OU-YANG ${ }^{1}$ \\ ${ }^{1}$ Department of Urology, The First Affiliated Hospital of Soochow University, Suzhou, Jiangsu 215006; \\ ${ }^{2}$ Department of Urology, Kunshan Chinese Medicine Hospital Affiliated to Nanjing University \\ of Chinese Medicine, Kunshan, Jiangsu 215300, P.R. China
}

Received February 21, 2020; Accepted September 16, 2020

DOI: 10.3892/ol.2020.12215

\begin{abstract}
The aim of the present study was to determine the effect of microRNA (miR)-155-5p on the expression of testican-1 (SPOCK1) and the invasion and migration of prostate cancer cells in vitro. Bioinformatics analysis and molecular biology assays revealed that SPOCK1 may be a direct target gene of miR-155-5p. In addition, a negative correlation was identified between SPCOK1 and miR-155-5p expression in prostate tumor tissues and cell lines. miR-155-5p mimic transfection inhibited SPOCK1 expression in PC3 cells and decreased cell migration and invasion abilities, while the expression of vimentin, $\mathrm{N}$-cadherin, E-cadherin, $\beta$-catenin, matrix metalloproteinase (MMP)3 and MMP9 was upregulated. In summary, SPOCK1 was found to be a target gene of miR155-5p in prostate cancer, and miR-155-5p acts as a tumor-suppressor gene and may inhibit SPOCK1-mediated prostate cancer progression.
\end{abstract}

\section{Introduction}

With a worldwide annual mortality of $>200,000$, prostate cancer has become the second leading cause of cancer-related mortality in men $(1,2)$. According to the recommended guidelines and current research, several biomarkers, such as prostate-specific antigen and prostate cancer antigen 3 , are used as screening indicators to detect patients at risk of developing prostate cancer or to monitor postoperative patients $(3,4)$. However, the pathogenesis of prostate carcinoma has yet to be fully elucidated. Further research on the development and progression of prostate cancer are key to reducing recurrence and mortality. Therefore, it is crucial to explore novel reliable biomarkers that are involved in the pathogenesis of prostate neoplasms.

Correspondence to: Professor Jun Ou-Yang, Department of Urology, The First Affiliated Hospital of Soochow University, 1 Shizi, Suzhou, Jiangsu 215006, P.R. China

E-mail: ouyangjun099@163.com

Key words: miR-155-5p, testican-1F, prostate cancer, migration
Testican-1 (SPOCK1) is a member of the secreted protein acidic and rich in cysteine family that encodes a matricellular $\mathrm{Ca}^{2+}$-binding glycoprotein and plays a key role in cell cycle regulation, apoptosis, DNA repair and metastasis (5-7). Due to its characteristic N-terminus, follistatin-like domain and C-terminus, SPOCK1 was found to be involved in cell proliferation, adhesion and migration (8). Recent studies reported that SPOCK1 is abnormally expressed in various tumors and is involved in glioblastoma cell invasion, hepatocellular carcinoma progression and the regulation of epithelial-to-mesenchymal transition (EMT) in lung cancer (9-11). In vitro and in vivo assays revealed that SPOCK1 promotes tumor growth and metastasis in human prostate cancer via several pathways, such as via PI3K/Akt, Wnt/catenin, Bcl-2 family and matrix metalloproteinases (MMPs) $(12,13)$. However, the mechanism underlying SPOCK1 overexpression remains unclear.

MicroRNAs (miRs/miRNAs), which are evolutionary conserved small non-coding RNAs, have been identified as regulators of gene expression and protein translation (14). Recent studies revealed different roles of miR-155-5p in various types of cancer (15-20). miR-155-5p was shown to be downregulated in gastric tumors (15), and overexpression of miR-155-5p inhibited the proliferation and promoted apoptosis of gastric cancer cell lines via decreasing mitogen-activated protein kinase 10 , while downregulation of miR-155-5p decreased cisplatin sensitivity (16). In addition, miR-155-5p was shown to suppress cell migration and invasion in the lung adenocarcinoma A549 cell line by targeting Smad2 (17). However, several studies have reported an opposite effect of miR-155-5p on other carcinomas. In colorectal carcinoma, miR-155-5p expression was upregulated and promoted the proliferation, invasion and metastasis of colorectal cancer cells (18). Increased miR-155-5p expression may also contribute to the suppression of tumor cell death in osteosarcoma (19). A recent study demonstrated that miR-155-5p contributes to EMT-associated oral squamous cell carcinoma (OSCC) progression and serves as a biomarker for predicting relapse, particularly for patients with early-stage OSCC (20).

Based on data obtained from TargetScan and miRBase, miR-155-5p appears to directly target SPOCK1 (Fig. 1A). Due to the carcinogenic function of SPOCK1 in prostate cancer, it was hypothesized that miR-155-5p may inhibit the 
invasion and migration of prostate cancer cells via targeting SPOCK1. However, to the best of our knowledge, few studies to date have investigated whether miR155-5p plays role in prostate tumorigenesis and cancer progression. Therefore, the aim of the present study was to investigate the expression of SPOCK1 and miR155-5p in 41 cases of prostate cancer using reverse transcription-quantitative PCR (RT-qPCR) analysis, evaluate the association between SPOCK1 and miR-155-5p in prostate cancer, and determine the role of miR-155-5p in the invasion and migration of prostate cancer cells in vitro.

\section{Materials and methods}

Patients and tissue samples. A total of 41 Chinese patients with prostate cancer who were admitted to Kunshan Hospital of Traditional Chinese Medicine between January, 2015 and December, 2018 were selected. Tumor tissues from each patient and matched adjacent non-tumor tissues were obtained with the patient's authorization. The mean age of the whole patient sample was $73.54 \pm 16.02$ years and the characteristics of all the patients are summarized in Table I. Total RNA was extracted to investigate the expression of SPOCK 1 and miR 155-5p. The protocol of the present study was approved by the Chinese Medicine Hospital Ethics Committee and all the patients provided written informed consent.

Data mining of datasets. Two datasets were selected from Oncomine (https://www.oncomine.org/): Tomlins Prostate (23 samples of normal prostate gland tissue, 13 samples of prostate intraepithelial neoplasia and 30 samples of prostate carcinoma) and Lapointe Prostate (41 samples of normal prostate gland tissue and 62 samples of prostate carcinoma). These datasets compared the expression of SPOCK1 between prostate cancer and normal tissues. Bioinformatics analysis using TargetScan and miRBase was conducted and SPOCK1 was identified as a direct target gene of miR-155-5p.

Cell lines and cell culture. The normal human prostate cell line RWPE-2 and four prostate cancer cell lines (PC3, 22Rv1, DU145 and LNCaP) were obtained from FuDan IBS Cell Center (Shanghai, China). The cells were cultured in corresponding media (RPMI-1640 medium for LNCaP, DU145 and RWPE-2 cells; F-12 medium for PC3 cells; and DMEM for 22Rv1 cells) supplemented with 10\% FBS (Gibco; Thermo Fisher Scientific, Inc.) and $1 \%$ antibiotic/antimycotic solution (Sigma-Aldrich; Merck $\mathrm{KGaA}$ ) at $37^{\circ} \mathrm{C}$ with $5 \% \mathrm{CO}_{2}$, according to the guidelines of the FuDan IBS Cell Center.

Cell transfection and luciferase reporter assay. PC3 cells were cultured in a 6-well plate and transfected with miR-155-5p mimic and miR-155-5p negative control (NC; Shanghai GenePharma Co., Ltd.) when the cells were $70 \%$ confluent. After 48 h, RT-qPCR was performed to evaluate transfection efficiency. SPOCK1 expression was evaluated using RT-qPCR and western blot assays to verify whether SPOCK1 is a direct target gene of miR-155-5p.

For the luciferase reporter assay, PC3 cells were seeded into 96-well plates at a density of $2 \times 10^{4}$ cells/well and grown to $70 \%$ confluence. Cells were co-transfected with
Table I. Characteristics of 41 patients with prostate cancer.

Characteristics

Age at diagnosis, years

$<70 \quad 16$

$\geq 70$

Baseline prostate-specific antigen level, ng/ml

$<4$

$4-9$

$\geq 10$

Clinical stage

T1c

T2

T3

Gleason score

miR-155-5p mimic or miR155-5p NC for $48 \mathrm{~h}$ at $37^{\circ} \mathrm{C}$, and SPOCK1-3'-untranslated region (UTR)-wild-type (WT) or SPOCK1-3'-UTR-mutant (MUT) plasmids (Shanghai GenePharma Co., Ltd.) using Lipofectamine ${ }^{\mathrm{TM}} 2000$ (Thermo Fisher Scientific, Inc.). A luciferase assay kit (BioLux ${ }^{\circledR}$ Gaussia; New England BioLabs, Inc.) was used to evaluate luciferase activity according to the manufacturer's protocol.

Western blot analysis. Total protein was extracted using RIPA buffer supplemented with PMSF (Beyotime Institute of Biotechnology). The samples were loaded in pre-cast protein electrophoresis gels (Thermo Fisher Scientific, Inc.) and were transferred to PVDF membranes (Beyotime Institute of Biotechnology). The following antibodies were used: Rabbit anti-human SPOCK1 polyclonal antibody (1:2,000; cat. no. ab229935; Abcam), rabbit anti-human MMP3 monoclonal antibody (1:2,000; cat. no. ab52915; Abcam), rabbit anti-human MMP9 polyclonal antibody (1:1,000; cat. no. ab38898; Abcam), mouse anti-human vimentin monoclonal antibody $(1: 1,000$; cat. no. ab8978; Abcam), rabbit anti-human $\beta$-catenin polyclonal antibody (1:6,000; cat. no. ab32572; Abcam), rabbit anti-human N-cadherin monoclonal antibody (1:1,000, cat. no. ab202030; Abcam), rabbit anti-human E-cadherin monoclonal antibody (1:1,000; cat. no. 3195; Cell Signaling Technology, Inc.) and rabbit anti-human GAPDH polyclonal antibody $(1: 1,000$; cat. no. 5174; Cell Signaling Technology, Inc.). The formula used to measure relative protein expression was as follows: Relative protein expression $=$ Grey value of each protein/grey value of GAPDH.

RT-qPCR analysis. Total RNA was extracted from cells or tissues using TRIzol ${ }^{\circledR}$ reagent (Thermo Fisher Scientific, Inc.) according to the manufacturer's protocol. RNA was reverse-transcribed into cDNA using the miScript II RT kit (Invitrogen; Thermo Fisher Scientific, Inc.). An iQ5 Real-Time PCR detection system (Bio-Rad Laboratories, Inc.) with 
A $\begin{array}{rrrr}\text { SPCOK1 WT: } 5^{\prime} & \text {...CACUUAAAGAUCCUUAGCAUUAA... } & 3^{\prime} \\ \text { I I I I I I I I } & & \\ \text { hsa-miR155-5p: } & \text { 3' } & \text { UGGGGAUAGUGCUAAUCGUAAUU } & 5^{\prime} \\ \text { SPCOK1 MUT: } & 5^{\prime} & \text {...CACUUAAAGAUCCUUGAUGCCGA... } & 3^{\prime}\end{array}$
B
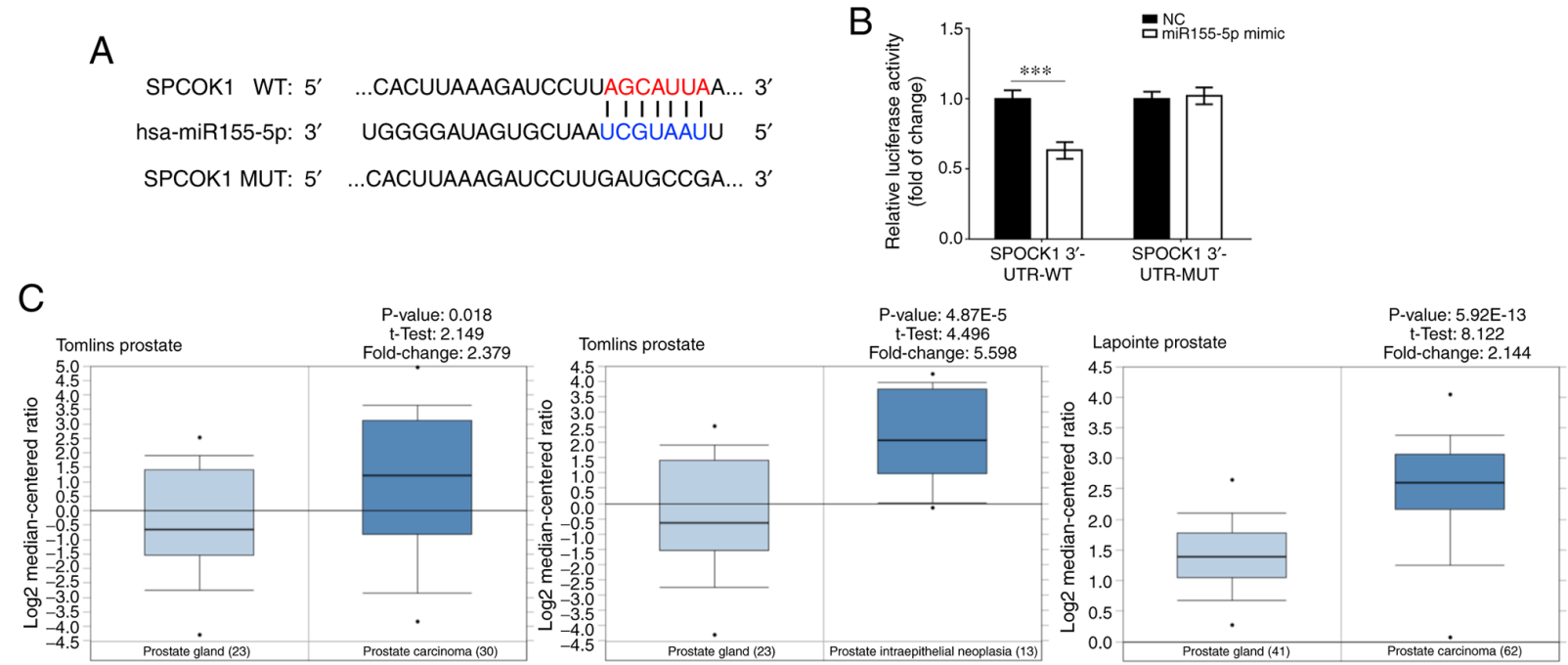

Figure 1. Results of public databases and luciferase reporter assay. (A) A WT-SPOCK1 and MUT-SPOCK1 luciferase reporter gene vector containing a 7-bp mutation on the predicted miR-155-5p binding site was constructed according to TargetScan and miRBase. (B) A luciferase reporter assay was conducted to confirm that SPOCK1 is a direct target of miR-155-5p. (C) Oncomine showed a higher expression of SPOCK1 in prostate carcinoma compared with that in corresponding non-tumor tissues. ${ }^{* * *} \mathrm{P}<0.001$. SPOCK1, testican-1; WT, wild-type; MUT, mutant; miR, microRNA.

the SYBR Premix Ex Taq ${ }^{\mathrm{TM}}$ kit (Takara Bio, Inc.) were used. The following primer pairs were used for the qPCR: miR-155-5p forward, 5'-UAAUACCGUCUUAAAACC GU-3' and reverse, 5'-UUCUGGGAACGUGAAACCT-3'; U6 forward, 5'-CGCTTCGGCAGCACATATACTAAA ATTGGAAC-3' and reverse, 5'-GCTTCACGAATTTGC GTGTCATCCTTGC-3'; SPOCK1 forward, 5'-AAGGGT CAAGCAGGAGGTCAT-3' and reverse, 5'-CACGAGGAT GCGAACAGAGTC-3'; and GAPDH forward, 5'-GAAGGT GAAGGTCGGAGT-3' and reverse, 5'-GAAGATGGTGAT GGGATTTC-3'. The following thermocycling conditions were used for the qPCR: $94^{\circ} \mathrm{C}$ for $4 \mathrm{~min}, 40$ cycles of $95^{\circ} \mathrm{C}$ for $1 \mathrm{~min}, 60^{\circ} \mathrm{C}$ for $1 \mathrm{~min}$ and $72^{\circ} \mathrm{C}$ for $1 \mathrm{~min}$. Subsequently, $2^{-\Delta \Delta \mathrm{Cq}}$ value/foldchangewasusedtocalculatetherelativeexpression $\left[\Delta \Delta \mathrm{Cq}=\left(\mathrm{Ct}_{\mathrm{rLV} \text {-SPARCL1-PGK-Puro-SAPRCL1 }} \mathrm{Cq}_{\mathrm{rLV}-\mathrm{SPARCL1} \text {-PGK-Puro-GAPDH }}\right)\right.$ $\left.\left(\mathrm{Cq}_{\text {Empty-Vector-SPARCL1 }}-\mathrm{Cq}_{\text {Empty-Vector-GAPDH }}\right)\right]$ (21).

Wound healing assay. To assess the role of miR-155-5p on the migration of PC3 cells, transfected cells were seeded in 6-well plates to complete confluence. The cell monolayer was scratched with a sterile plastic $200-\mu 1$ micropipette tip and PBS (Gibco; Thermo Fisher Scientific, Inc.) was used to remove loose cells. Subsequently, serum-free Opti-MEM (Gibco; Thermo Fisher Scientific, Inc.) was added to the wells. Following incubation at $37^{\circ} \mathrm{C}$ with $5 \% \mathrm{CO}_{2}$ for $24 \mathrm{~h}$, images were captured using an inverted microscope (magnification x40, IX73; Olympus Corporation). The following formula was used: Residual wound area rate (relative proportion of the wound $)=($ width of the wound/initial width on day 0$) \times 100 \%$.

Transwell assay. Transwell culture inserts (pore size, $8 \mathrm{~mm}$; Guangzhou Jet Bio-Filtration Co., Ltd.) were placed into the wells of 24-well plates to separate upper and lower chambers. The upper side of the membrane was precoated with Matrigel and incubated at $37^{\circ} \mathrm{C}$ for $1 \mathrm{~h}$ for gel formation. Subsequently, F-12 medium was added to the lower chamber, whereas $1 \times 10^{5}$ cells/well in Opti-MEM were added to the upper chamber. After $72 \mathrm{~h}$ of incubation at $37^{\circ} \mathrm{C}$, the number of invading cells was counted using a counting chamber under an inverted light microscope (magnification x100; IX73; Olympus Corporation).

Statistical analysis. Continuous variables are expressed as the mean \pm SD and analyzed with an unpaired t-test. Pearson's correlation test was used to analyze the relationship between miR-155-5p and SPOCK1 expression in prostate tumors. The F-test with Tukey's test was used to compare relative expression levels of miR-155-5p and SPOCK1 mRNA in RWPE-2 and prostate cancer cell lines. All data were analyzed using SPSS 20.0 (IBM, Corp.) or GraphPad Prism 6.0 (GraphPad Software, Inc.). $\mathrm{P}<0.05$ was considered to indicate a statistically significant difference.

\section{Results}

Expression levels and correlation analysis of miR-155-5p and SPOCK1 in prostate cancer. Based on TargetScan and miRBase analysis, miR-155-5p was found to directly target SPOCK1. A WT-SPOCK1 and MUT-SPOCK1 luciferase reporter gene vector containing a 7-bp mutation on the predicted miR-155-5p binding site was constructed (Fig. 1A). The luciferase reporter assay revealed that luciferase activity was decreased in the SPOCK1 3'-UTR WT group transfected with miR-155-5p mimic, thus suggesting that SPOCK1 may be a direct target gene of miR-155-5p (Fig. 1B).

SPOCK1 gene expression was found to be significantly higher in prostate carcinoma compared with that in corresponding non-tumor tissues (Figs. 1C and 2A). Furthermore, miR-155-5p expression in prostate tumor tissues was lower compared with that in normal tissues and significantly lower compared with SPOCK1 expression in prostate tumor tissues (Fig. 2B and C). In addition, there was a negative correlation between the expressions of SPOCK1 and miR-155-5p in prostate tumors (Fig. 2D). 

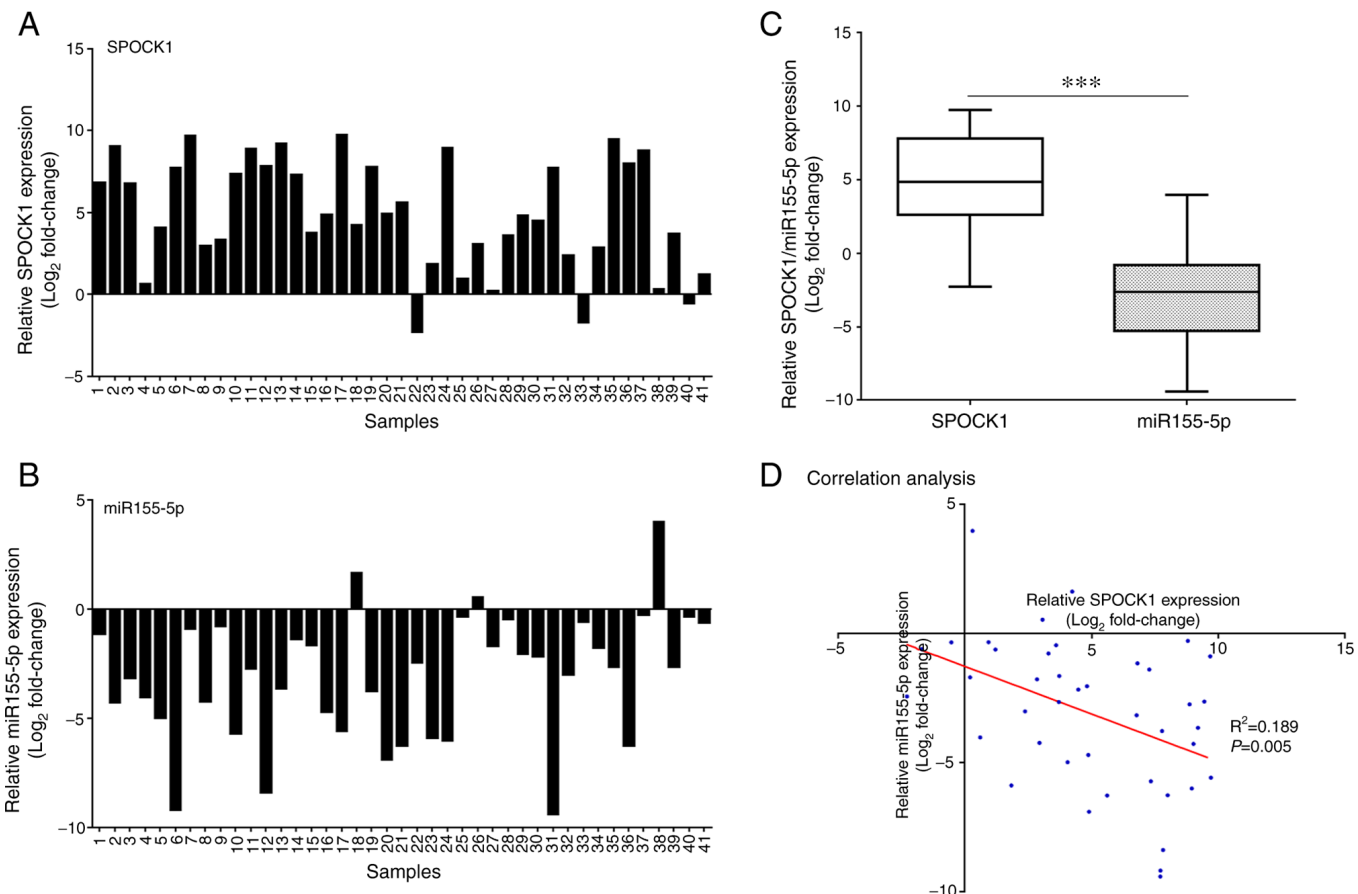

Figure 2. Association between SPOCK1 and miR-155-5p expression in prostate carcinoma. Relative expression of (A) SPOCK1 and (B) miR-155-5p in prostate carcinoma. (C) miR-155-5p expression in prostate tumors was significantly lower compared with SPOCK1 expression. (D) A negative correlation was observed between SPOCK1 and miR-155-5p expression in prostate tumors. ${ }^{* * *} \mathrm{P}<0.001$. SPOCK1, testican-1; miR, microRNA
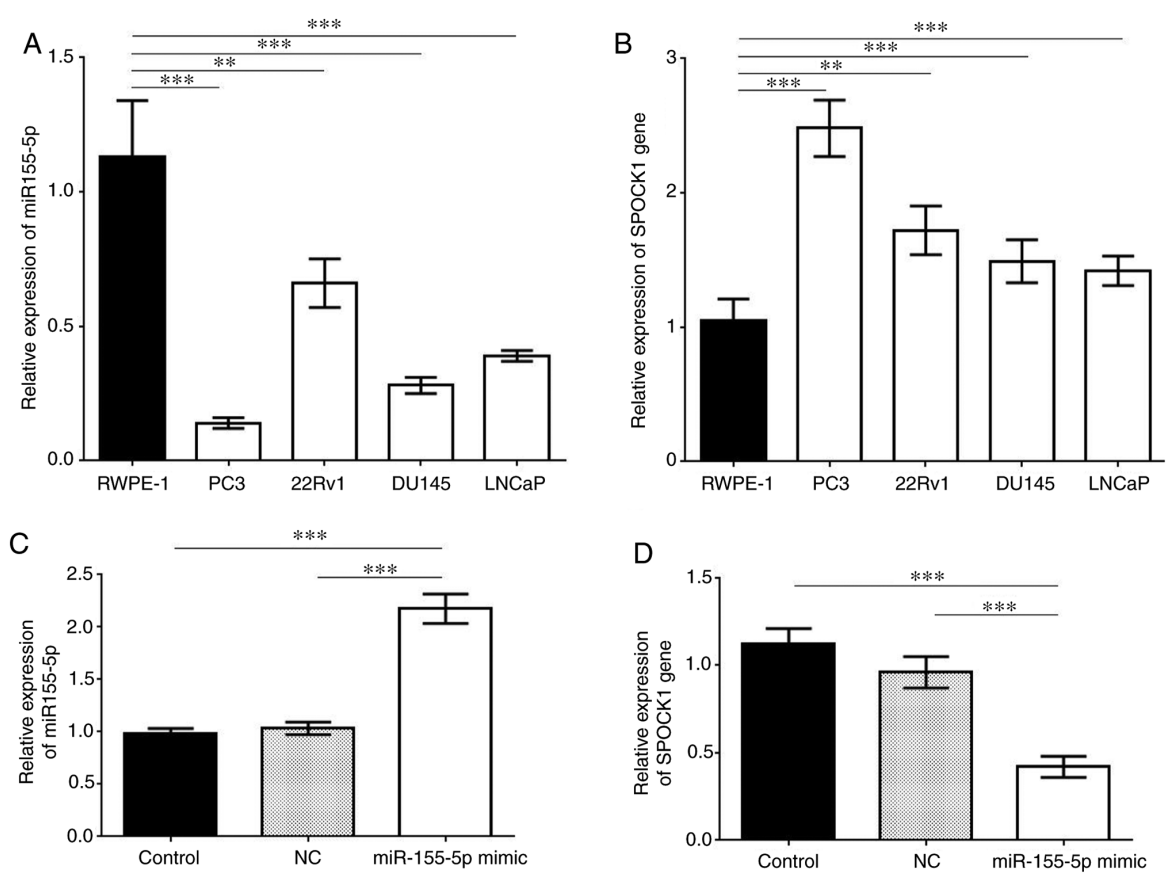

E

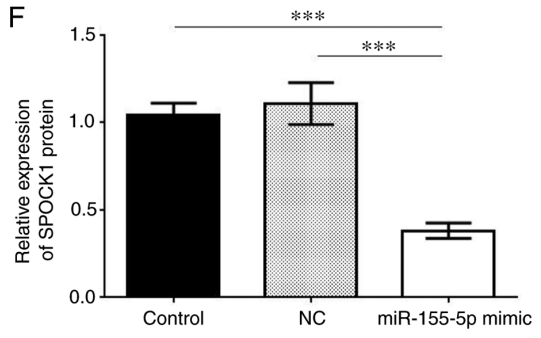

Figure 3. miR-155-5p inhibits SPOCK1 in RWPE-1 and prostate cancer cell lines. mRNA expression of (A) miR-155-5p and (B) SPOCK1 in RWPE-1 cells and prostate cancer cell lines. Regulation of (C) miR-155-5p and (D) SPOCK1 mRNA expression in PC3 cells using miR-155-5p mimic. (E) Western blot analysis showing decreased SPOCK1 protein expression following miR-155-5p mimic transfection. (F) Relative expression of SPOCK1 protein. ${ }^{* *} \mathrm{P}<0.01$ and ${ }^{* * * *} \mathrm{P}<0.001$. SPOCK1, testican-1; miR, microRNA. 

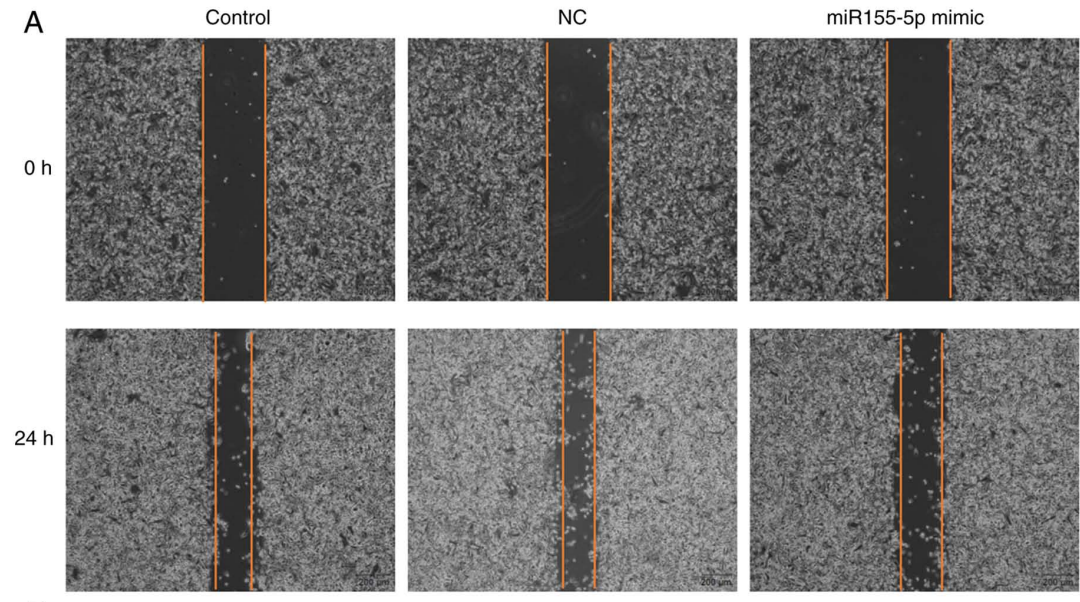

B

NC

miR155-5p mimic
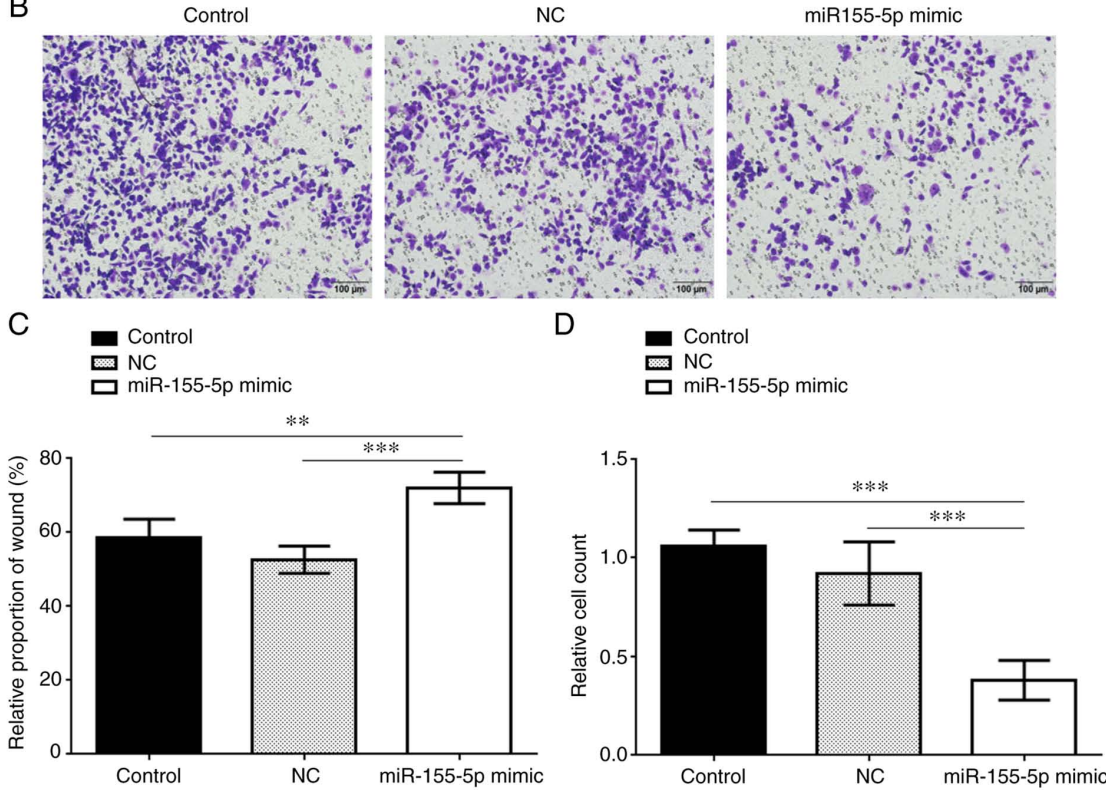

Figure 4. Effects of miR-155-5p mimic on the invasion and migration of PC3 cells. (A) Wound healing assay. (B) Transwell assay. (C) Relative proportion of wound. (D) Relative cell count on Transwell assay. ${ }^{* *} \mathrm{P}<0.01$ and ${ }^{* * *} \mathrm{P}<0.001 . \mathrm{miR}$, microRNA.
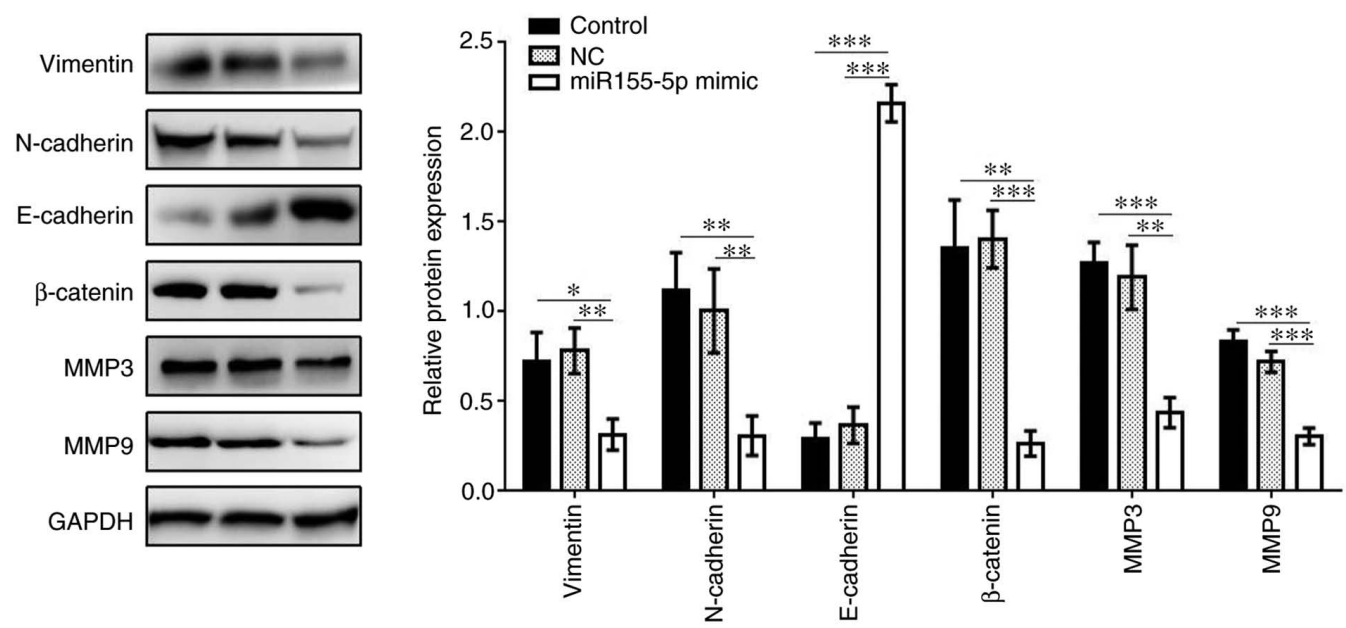

Figure 5. Effects of miR-155-5p mimic on the expression of the invasion-associated proteins vimentin, N-cadherin, E-cadherin, $\beta$-catenin, MMP3 and MMP9. ${ }^{*} \mathrm{P}<0.05 ;{ }^{* *} \mathrm{P}<0.01$ and ${ }^{* * *} \mathrm{P}<0.001$. miR, microRNA; MMP, matrix metalloproteinase. 
miR-155-5p, while the expression levels of SPOCK1 in these cancer cell lines were significantly higher $(\mathrm{P}<0.01$ or $\mathrm{P}<0.001$; Fig. 3A and B). PC3 cells exhibited the lowest miR-155-5p and highest SPOCK1 expression levels. When miR-155-5p was upregulated by $\mathrm{miR}-155-5 \mathrm{p}$ mimic, the relative expression of SPOCK1 markedly decreased compared with that in the control groups, as shown by both RT-qPCR and western blot assays (Fig. 3). Therefore, SPOCK1 was suppressed by miR-155-5p in PC3 cells due to their specific binding site.

Overexpression of $\mathrm{miR}-155-5 \mathrm{p}$ suppresses invasion and migration of PC3 cells. Cell migration and invasion were measured by scratch and Transwell assays, respectively, and were both found to be significantly inhibited in the miR-155-5p mimic group compared with the control groups (Fig. 4). In addition, the expression levels of vimentin, N-cadherin, $\beta$-catenin, MMP3 and MMP9 were all markedly decreased in the miR-155-5p mimic group compared with those in the control groups, while the expression of E-cadherin was upregulated in the miR-155-5p mimic group (Fig. 5). Therefore, miR-155-5p may inhibit the invasion and migration ability of PC3 cells via downregulating the expression of SPOCK1 and its downstream regulators.

\section{Discussion}

SPOCK1 is a matricellular $\mathrm{Ca}^{2+}$-binding glycoprotein that has a characteristic $\mathrm{N}$-terminus, follistatin-like domain and C-terminus, which plays a key role in cancer metastasis. SPOCK1 expression was found to be significantly higher in prostate carcinoma compared with that in the normal prostate gland by both Oncomine data and the results of the present study. In previous studies, SPOCK1 was identified as a novel oncogene with critical effects on the PI3K/Akt and Wnt/ $\beta$-catenin pathways, the Bcl-2 family and MMPs $(12,13,22)$.

Since SPOCK1 plays a role in the tumorigenesis and progression of prostate cancer, the present study investigated the inhibitory effect of an upstream regulator, miR-155-5p, on the invasion and migration of prostate cancer cells in vitro. Although previous research reported the carcinogenic role of miR-155-5p in colorectal carcinoma, osteosarcoma and oral squamous cell carcinoma (18-20), recent studies demonstrated that miR-155-5p inhibited cell proliferation and promoted apoptosis in gastric cancer cell lines via decreasing mitogen-activated protein kinase 10 , while downregulation of miR-155-5p decreased cisplatin sensitivity $(15,16)$. In addition, miR-155-5p suppressed the migration and invasion of the lung adenocarcinoma A549 cell line by targeting Smad2 (17). In a previous study, promoter-associated $\mathrm{CpG}$ islands of miR-155 were frequently hypermethylated in prostate cancer, but not in benign prostatic hyperplasia samples (23). Due to methylation, highly significant downregulation of miR-155-5p was detected in prostate cancer compared with benign samples (23). In the present study, RT-qPCR analysis demonstrated that the expression of $\mathrm{miR}-155-5 \mathrm{p}$ in prostate tumor tissues was also higher compared with that in normal prostate tissues. Furthermore, another study focused on chronic non-bacterial prostatitis (CNP) and revealed that rno-miR-155-5p levels in rat prostate samples of the carrageenan injection group were significantly upregulated compared with the blank control (without any interference) or normal saline injection groups, which may prove to be of value for identifying novel mechanisms of action of miRNAs in immune regulation and effective target-specific theragnosis of CNP (24). Therefore, the present study demonstrated that decreased levels of miR-155-5p were involved in prostate tumorigenesis and cancer progression.

In the present study, SPOCK1 was identified as a target gene of miR-155-5p in PC3 cells via bioinformatics analysis, luciferase reporter assays, RT-qPCR analysis and western blotting. RT-qPCR also revealed the presence of a negative association between SPOCK1 and miR-155-5p in prostate tumor tissues and cell lines. This indicated that miR-155-5p downregulation may play a role in SPOCK1-mediated prostate cancer progression.

EMT plays a key role in cancer metastasis, particularly in prostate neoplasms (25). Recent studies demonstrated that SPOCK1 regulates the EMT process during cancer metastasis $(12,13,26)$. MMP3 and MMP9 are two mesenchymal markers that promote EMT and distant metastasis (27). In a previous study, SPOCK1 knockdown in PC3 cells significantly inhibited cell invasion and migration via downregulation of MMP3 and MMP9 (12). In addition, EMT and metastasis of prostate cancer were also regulated by the $\mathrm{Wnt} / \beta$-catenin signaling pathway, which was found to be aberrantly activated in prostate cancer (28). Therefore, the present study investigated the expression of MMP3, MMP9 and key proteins of the $\mathrm{Wnt} / \beta$-catenin signaling pathway to elucidate the potential molecular mechanisms underlying the effect of miR-155-5p on the PC3 cell line. Upon miR-155-5p mimic transfection, the protein levels of vimentin, $\beta$-catenin, $\mathrm{N}$-cadherin, MMP3 and MMP9 were decreased, while the protein levels of E-cadherin were increased. These results indicated that miR-155-5p could exert its tumor-suppressor role by suppressing MMP3 and MMP9 expression and modulating the $\mathrm{Wnt} / \beta$-catenin signaling pathway.

In conclusion, the present study demonstrated that the oncogene SPOCK1 is a target gene of miR-155-5p in prostate cancer. SPOCK1 expression was found to be negatively correlated with that of miR-155-5p in both prostate tumor tissues and cell lines. In addition, miR-155-5p suppressed the invasion and migration of PC3 cells by decreasing the expression of MMPs and regulating the $\mathrm{Wnt} / \beta$-catenin signaling pathway. The findings of the present study suggest that miR-155-5p acts as a tumor suppressor gene in prostate carcinoma, inhibits SPOCK1-mediated prostate cancer progression, and may be a valuable candidate for targeted therapy.

\section{Acknowledgements}

Not applicable.

\section{Funding}

This study was supported by the Kunshan Science and Technology Program of Social Development (grant no. KS1834).

\section{Availability of data and materials}

The datasets used and/or analyzed during the current study are available from the corresponding author on reasonable request. 


\section{Authors' contributions}

LYY and JOY conceived and planned the study. LYY, JM, XMZ and JOY performed the experiments. LYY and JM analyzed the data. All authors read and approved the manuscript and agreed to be accountable for all aspects of the research in ensuring that the accuracy or integrity of any part of the work are appropriately investigated and resolved.

\section{Ethics approval and consent to participate}

The protocol of the present study was approved by the Chinese Medicine Hospital Ethics Committee and all the patients provided written informed consent.

\section{Patient consent for publication}

Not applicable.

\section{Competing interests}

All the authors declare that they have no competing interests.

\section{References}

1. Siegel RL, Miller KD and Jemal A: Cancer statistics, 2018 CA Cancer J Clin 68: 7-30, 2018.

2. Cao L, Lee CH, Ning J, Handy BC, Wagar EA and Meng QH: Combination of prostate cancer antigen 3 and prostate-specific antigen improves diagnostic accuracy in men at risk of prostate cancer. Arch Pathol Lab Med 14: 1106-1112, 2018.

3. Nordström T, Akre O, Aly M, Grönberg $\mathrm{H}$ and Eklund $\mathrm{M}$ : Prostate-Specific antigen (PSA) density in the diagnostic algorithm of prostate cancer. Prostate Cancer Prostatic Dis 21: 57-63, 2018.

4. Chunhua L, Zhao H, Zhao H, Lu Y, Wu J, Gao Z, Li G, Zhang Y and Wang K: Clinical significance of peripheral blood PCA3 gene expression in early diagnosis of prostate cancer. Transl Oncol 11: 628-632, 2018

5. Bradshaw AD and Sage EH: SPARC, a matricellular protein that functions in cellular differentiation and tissue response to injury. J Clin Invest 107: 1049-1054, 2001.

6. Ma LJ, Wu WJ, Wang YH, Wu TF, Liang PI, Chang IW, He HL and Li CF: SPOCK1 overexpression confers a poor prognosis in urothelial carcinoma. J Cancer 7: 467-476, 2016.

7. Dhamija R, Graham JM Jr, Smaoui N, Thorland E and Kirmani S: Novel de novo SPOCK1 mutation in a proband with developmental delay, microcephaly and agenesis of corpus callosum. Eur J Med Genet 57: 181-184, 2014.

8. Song X, Han P, Liu J, Wang Y, Li D, He J, Gong J, Li M, Tu W, Yan W, et al: Up-Regulation of SPOCK1 induces epithelial-mesenchymal transition and promotes migration and invasion in esophageal squamous cell carcinoma. J Mol Histol 46: 347-356, 2015.

9. Li Y, Chen L, Chan TH, Liu M, Kong KL, Qiu JL, Li Y, Yuan YF and Guan XY: SPOCK1 is regulated by CHD1L and blocks apoptosis and promotes HCC cell invasiveness and metastasis in mice. Gastroenterology 144: 179-191, 2013.

10. Colin C, Baeza N, Bartoli C, Fina F, Eudes N, Nanni I, Martin PM, Ouafik L and Figarella-Branger D: Identification of genes differentially expressed in glioblastoma versus pilocytic astrocytoma using suppression subtractive hybridization. Oncogene 25: 2818-2826, 2006.

11. Miao L, Wang Y, Xia H, Yao C, Cai H and Song Y: SPOCK1 is a novel transforming growth factor-beta target gene that regulates lung cancer cell epithelial-mesenchymal transition. Biochem Biophys Res Commun 440: 792-797, 2013.
12. Chen Q, Yao YT, Xu H, Chen YB, Gu M, Cai ZK and Wang Z: SPOCK1 promotes tumor growth and metastasis in human prostate cancer. Drug Des Devel Ther 10: 2311-2321, 2016.

13. Yang C, Fischer-Kešo R, Schlechter T, Ströbel P, Marx A and Hofmann I: Plakophilin 1-deficient cells upregulate SPOCK1: Implications for prostate cancer progression. Tumour Biol 36: 9567-9577, 2015.

14. Torrini C, Cubero RJ, Dirkx E, Braga L, Ali H, Prosdocimo G, Gutierrez MI, Collesi C, Licastro D, Zentilin L, et al: Common regulatory pathways mediate activity of MicroRNAs inducing cardiomyocyte proliferation. Cell Rep 27: 2759-2771, 2019.

15. Li H, Xie S, Liu M, Chen Z, Liu X, Wang L, Li D and Zhou Y: The clinical significance of downregulation of mir-124-3p, mir-146a-5p, mir-155-5p and mir-335-5p in gastric cancer tumorigenesis. Int J Oncol 45: 197-208, 2014.

16. Li S, Zhang T, Zhou X, Du Z, Chen F, Luo J and Liu Q: The tumor suppressor role of miR-155-5p in gastric cancer: The tumor suppressor role of miR-155-5p in gastric cancer. Oncol Lett 16: 2709-2714, 2018.

17. Lin J, Chen Y, Liu L, Shen A and Zheng W: MicroRNA-155-5p suppresses the migration and invasion of lung adenocarcinoma A549 cells by targeting smad2. Oncol Lett 16: 2444-2452, 2018.

18. Qu YL, Wang HF, Sun ZQ, Tang Y, Han XN, Yu XB and Liu K: Up-Regulated miR-155-5p promotes cell proliferation, invasion and metastasis in colorectal carcinoma. Int J Clin Exp Pathol 8: 6988-6994, 2015.

19. Bhattacharya S, Chalk AM, Ng AJ, Martin TJ, Zannettino AC, Purton LE, Lu J, Baker EK and Walkley CR: Increased miR-155-5p and reduced miR-148a-3p contribute to the suppression of osteosarcoma cell death. Oncogene 35: 5282-5294, 2016.

20. Kim H, Yang JM, Ahn SH, Jeong WJ, Chung JH and Paik JH: Potential oncogenic role and prognostic implication of microRNA-155-5p in oral squamous cell carcinoma. Anticancer Res 38: 5193-5200, 2018.

21. Livak KJ and Schmittgen TD: Analysis of relative gene expression data using real-time quantitative PCR and the 2(-Delta Delta C(T)) method. Methods 25: 402-408, 2001.

22. Liu YZ, Guo YF, Wang L, Tan LJ, Liu XG, Pei YF, Yan H, Xiong DH, Deng FY, Yu N, et al: Genome-Wide association analyses identify SPOCK as a key novel gene underlying age at menarche. PLoS Genet 5: e1000420, 2009.

23. Daniunaite K, Dubikaityte M, Gibas P, Bakavicius A, Lazutka JR, Ulys A, Jankevicius F and Jarmalaite S: Clinical significance of miRNA host gene promoter methylation in prostate cancer. Hum Mol Genet 26: 2451-2461, 2017.

24. Zhang L, Liu Y, Chen XG, Zhang Y, Chen J, Hao ZY, Fan S, Zhang LG, Du HX and Liang CZ: MicroRNA expression profile in chronic nonbacterial prostatitis revealed by next-generation small RNA sequencing. Asian J Androl 21: 351-359, 2019.

25. Chen WY, Tsai YC, Yeh HL, Suau F, Jiang KC, Shao AN, Huang J and Liu YN: Loss of SPDEF and gain of TGFBI activity after androgen deprivation therapy promote EMT and bone metastasis of prostate cancer. Sci Signal 10: 492, 2017

26. Huber MA, Azoitei N, Baumann B, Grünert S, Sommer A, Pehamberger $\mathrm{H}$, Kraut N, Beug $\mathrm{H}$ and Wirth T: NF-kappaB is essential for epithelial-mesenchymal transition and metastasis in a model of breast cancer progression. J Clin Invest 114: 569-581, 2004.

27. Yang H, Liang J, Zhou J, Mi J, Ma K, Fan Y, Ning J, Wang C, Wei $\mathrm{X}$ and Li E: Knockdown of RHOC by shRNA suppresses invasion and migration of cholangiocellular carcinoma cells via inhibition of MMP2, MMP3, MMP9 and epithelial-mesenchymal transition. Mol Med Rep 13: 5255-5261, 2016

28. Chen L, Mai W, Chen M, Hu J, Zhuo Z, Lei X, Deng L, Liu J, Yao N, Huang M, et al: Arenobufagin inhibits prostate cancer epithelial-mesenchymal transition and metastasis by down-regulating $\beta$-catenin. Pharmacol Res 123: 130-142, 2017.

This work is licensed under a Creative Commons Attribution-NonCommercial-NoDerivatives 4.0 International (CC BY-NC-ND 4.0) License. 